\title{
Lactancia Materna Exclusiva: Expectativa versus Realidad
}

\author{
Exclusive Breastfeeding: Expectations vs. Reality
}

\section{Sandra Carolina Gale* Karen Erazo**}

\section{RESUMEN}

Antecedentes y Objetivo: La lactancia materna es ampliamente reconocida como el mejor alimento para bebés, la Organización Mundial de la Salud (OMS) recomienda que los niños reciban lactancia materna exclusiva durante los primeros seis meses de vida, seguida de una combinación de lactancia materna continua y una alimentación apropiada hasta los 2 años de edad. Objetivo: Esta investigación tiene como objetivo conocer cuál fue el tipo de lactancia recibida en los niños menores de 18 meses y analizar los principales factores que favorecen y/o interfieren en la lactancia materna exclusiva e identificar la edad en que iniciaron la ablactación. Pacientes y métodos: Estudio descriptivo. Se aplicó una encuesta a madres con lactantes menores de 18 meses ingresados en la Sala de Lactantes Hospital Nacional Mario Catarino Rivas, HNMCR. Resultados: De las madres que participaron en este estudio $40 \%$ brindó lactancia materna exclusiva manteniéndose como principal causa de su destete la hipogalactia. El inicio de la ablactación se produjo a los 6 meses de edad en un $56 \%$. Conclusiones: Es necesario incrementar las acciones de salud con vistas a fortalecer los factores que promuevan la lactancia materna y disminuir aquellos que favorecen a un destete temprano.

\section{PALABRAS CLAVE}

Lactancia Materna, destete, métodos de alimentación.

\section{ABSTRACTS}

Background and Objectives: Breastfeeding is widely recognized as the best food for babies. The World Health Organization (WHO) recommends that children receive exclusive breastfe-

\footnotetext{
*Médico Residente Tercer Año de Pediatría, Universidad Nacional Autónoma de Honduras en el Valle de Sula UNAH-VS **Coordinadora del Postgrado de Pediatría UNAH-VS Dirigir correspondencia a: carolina_gale@hotmail.com Recibido: 16/Jun/2015 Aprobado:09/Jul/2015
}

eding for the first six months of life, followed by a combination of continued breastfeeding and adequate food up to 2 years old. Objective: This research aims to determine what was the type of feeding received in children under 18 months and analyze the main factors that promote and/ or interfere with exclusive breastfeeding and identify the age at which weaning began. Patients and Methods: In this descriptive study, a survey was applied to mothers with infants under 18 months admitted at the Toddler ward-Hospital Nacional Mario Catarino Rivas, HNMCR. Results: $40 \%$ of mothers that participated in this study gave exclusive breastfeeding. The main cause of weaning was hypogalactia, the onset of weaning occurred at 6 months of age in $56 \%$ of cases. Conclusions: It is necessary to increase health activities in order to strengthen the protective factors of breastfeeding and eliminate the most common cause of weaning.

\section{KEY WORDS}

Breastfeeding, weaning, feeding methods.

\section{INTRODUCCIÓN}

La leche materna constituye el mejor alimento que puede darse en forma exclusiva a un niño hasta los seis meses de edad y con otros alimentos hasta los dos años, ${ }^{(1)}$ ya que contribuye de forma eficaz a su desarrollo físico, intelectual y social, ${ }^{(2)}$ impactando en causas evitables de morbi-mortalidad infantil, sin distinción de nivel socioeconómico o educación de la madre. ${ }^{(3)}$

Los beneficios de la lactancia han sido bien documentados; disminuye la morbilidad de las enfermedades diarreicas, respiratorias, alérgi$\mathrm{cas}^{\left({ }^{(4)}\right.}$ aporte de células vivas, enzimas digestivas, inmunomoduladores y factores de crecimiento. ${ }^{(5)}$

Según la OMS, solo un 35\% de los lactantes de todo el mundo son alimentados exclusivamen- 
te con leche materna durante los primeros cuatro meses de vida. ${ }^{(6)}$

Aunque existe muy limitada información nacional sobre las causas de destete $\operatorname{precoz}^{(7)}$ variados factores socioeconómicos y culturales influyen en el abandono de la lactancia materna, ${ }^{(8)}$ entre la principal causa es que la madre o el lactante experimentan insatisfacción o dificultad con el proceso de lactancia materna ${ }^{(9)}$ sumado a la gran difusión de fórmulas lácteas por parte de la industria, desconocimiento de técnicas de amamantamiento, la introducción temprana de otros alimentos, la incorporación de la mujer al ambiente laboral $^{(10)}$ y la falta de apoyo para desarrollar exitosamente la práctica del amamantamiento. ${ }^{(11)}$

Las madres adolescentes, tienen menor probabilidad de dar lactancia materna debido a dos factores: escaso nivel educativo y bajo nivel socioeconómico. ${ }^{(10,12)}$

El objetivo del presente estudio fue conocer el tipo de lactancia recibida y analizar los principales factores que favorecen y/o que interfieren en la lactancia materna exclusiva de los niños menores de 18 meses ingresados en la Sala de Lactantes del HMCR, durante el mes de mayo del 2015.

\section{PACIENTES Y MÉTODOS}

El estudio se realizó en el Hospital Mario Catarino Rivas (HNMCR), de la ciudad de San Pedro Sula, Honduras. Se incluyeron 30 madres y a sus hijos ingresado en sala de lactantes durante el mes de mayo del 2015; se les aplicó un instrumento tipo encuesta que constaba de 20 preguntas cerradas previo consentimiento informado. Las variables del instrumento fueron la edad de la madre, escolaridad, ocupación, estado civil, tipo de parto, hospitalización de recién nacido, conocimiento sobre lactancia materna, edad de introducción de la formula, tipo de agua para la preparación del biberón, enfermedades gastro- intestinales asociadas y edad de ablactación.

Los criterios de exclusión fueron los siguientes:
- Lactante mayor de 18 meses de edad

- Madres que no quisieron participar

Se analizaron los datos con frecuencia, porcentajes y la tabulación de datos se hizo a computadora con Epi info.

\section{RESULTADOS:}

Se incluyeron en el estudio a 30 madres que tenían un hijo menor de 18 meses hospitalizado en la Sala de Lactantes cuyas edades de hijos estaba distribuida 14 (47\%) entre 0-6 meses; 14 (47\%) entre 6-12 meses y 2 (6\%) entre 12-18 meses de edad.

El tipo de lactancia recibida fué 13 (43\%) lactancia mixta (materna y formula de inicio), 12 (40\%) lactancia materna exclusiva y 5 (17\%) formula de inicio. Ver gráfico No.1

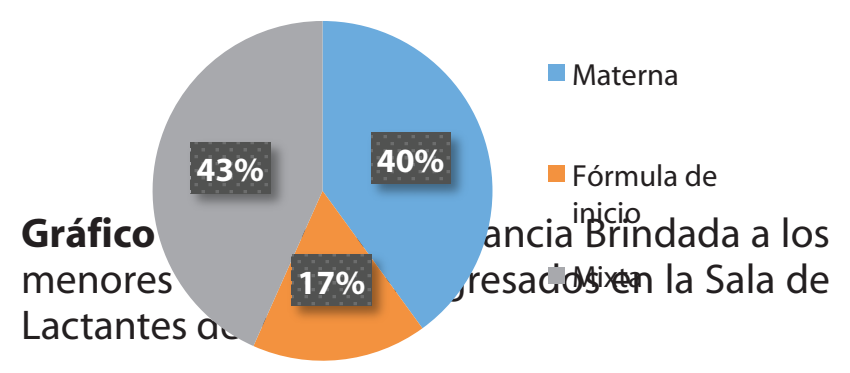

Se encontró que de las 30 madres 6 eran adolescentes, 4 (66\%) tenían 17 años, 1 (17\%) de 16 años y 1 (17\%) de 15 años; y de estas el 50\% prefiere dar alimentación con formula a sus hijos lactantes. Ver gráfico No. 2

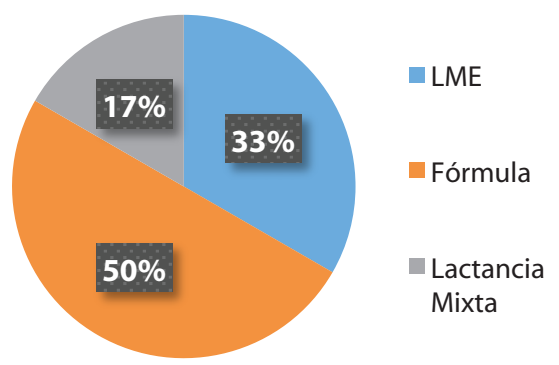

Gráfico No. 2 Tipo de alimentación brindada por madres adolescentes de los menores de 18 meses de edad de la Sala de Lactantes HNMCR. 
El tipo de parto de los niños incluidos en el estudio fue de 22 (73\%) vaginales y 8 (27\%) por cesárea; 8 (36\%) de las pacientes que dieron a luz por parto vaginal y $3(37 \%)$ pacientes que parieron por cesárea brindaron lactancia materna exclusiva.

Un total de 8 (27\%) pacientes fueron hospitalizados al nacer, de los cuales a $6(75 \%)$ se les introdujo formula de inicio después de su egreso hospitalario.

El principal motivo de dar lactancia materna exclusiva fue de 18 (72\%) por sus beneficios, 5 (20\%) por recomendación de medico durante el embarazo.

El conocimiento sobre los beneficios brindados por la lactancia materna, 14 (47\%) de las encuestadas desconocían algún tipo de beneficio y un 8 (27\%) mencionó la protección contra enfermedades. Ver cuadro No.1.

Tabla No.1 Conocimiento sobre los beneficios de la Lactancia Materna en las madres de los menores de 18 meses ingresados en la Sala de lactantes del HNMCR.

\begin{tabular}{lcc}
\hline $\begin{array}{l}\text { Conocimiento } \\
\text { sobre LM }\end{array}$ & $\mathrm{n}=30$ & $\%$ \\
\hline No sabe & & \\
beneficios & 14 & $47 \%$ \\
Inmunidad & 8 & $27 \%$ \\
Vitaminas & 4 & $13 \%$ \\
Crecimiento & 4 & $13 \%$ \\
\hline LM: lactancia Materna &
\end{tabular}

Sobre el significado de lactancia materna exclusiva 29 (97\%) madres desconocían de que se trataba y nunca habían escuchado hablar de ella.

De los 18 niños que no recibieron lactancia materna exclusiva, la principal causa referida para el inicio de formula láctea fue 9 (50\%) por haber tenido problemas para lactar con LM y $4(22 \%)$ por indicación médica y $3(17 \%)$ porque trabajaban. La edad a la que se introdujo la formula láctea fue de 9 (50\%) entre 1-3 meses de edad y 7 (39\%) antes de cumplir el mes de edad.

Ver gráfico No. 3.

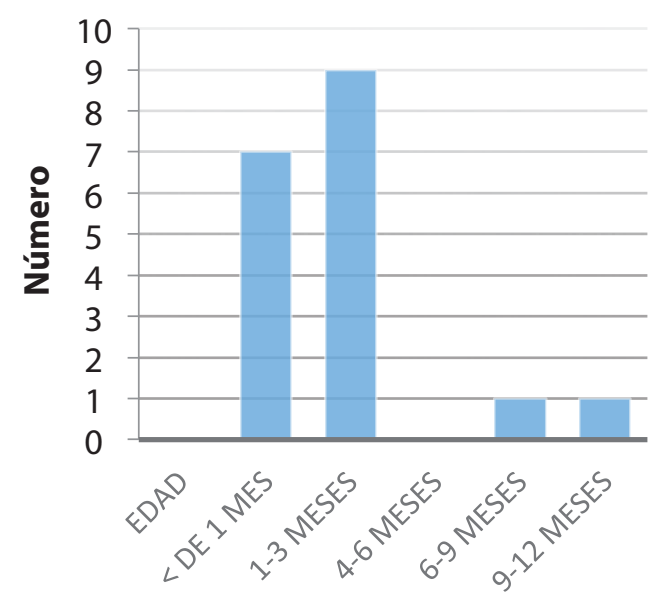

Gráfico No. 3 Edad de inicio de formula láctea en los menores de 18 meses de la Sala de Lactantes HNMCR.

De los 12 niños alimentados con lactancia materna exclusiva 7 (58\%) presentaron cuadros gastrointestinales pero ninguno requirió hospitalización, sin embargo al iniciar alimentación mixta el 50\% amerito hospitalización posterior por cuadro entérico. Ver gráfico No. 4.

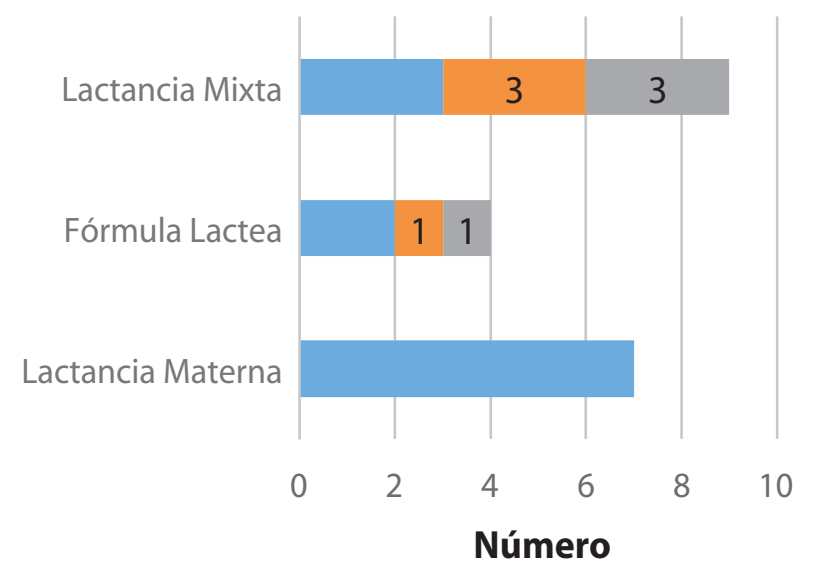

Enf. Gastro $\quad$ Hospitaliz. $\quad$ Agua Hervida

Gráfico No. 4 Comparación entre el tipo de lactancia administrada y la incidencia de enfermedades gastrointestinales, necesidad de hospitalización y tipo de agua utilizada en los lactantes menores de 18 meses de edad. 
Un total de 11 (92\%) madres que brindaban lactancia materna exclusiva no salen de su casa sin llevar al lactante ya que no saben de qué manera pueden alimentarlo si no están con ellos.

En 16 niños, ya se había realizado la ablactación; de ellos, en 9(56\%), fue a los 6 meses, en 4(25\%) a los 5 meses. Ver gráfica No 5.



Gráfico No. 5 Edad de inicio ablactación en los menores de 18 meses de la Sala de Lactantes HNMCR.

\section{DISCUSIÓN:}

Existen pocos datos nacionales sobre las causas del destete precoz, a pesar de la amplia preocupación que ha habido sobre el tema en los últimos 20 años.

Datos confirman que al menos dos de cada diez niños dejan de ser alimentados con lactancia materna exclusiva al mes de vida pese a las recomendaciones de la OMS y de la Academia Americana de Pediatría, ${ }^{(7)}$ esto lo observamos en nuestro estudio ya que en 7 (23\%) de los 30 niños se introdujo la formula antes de cumplir su primer mes, ofrecida en su mayoría como alimentación complementaria a la lactancia materna ya que en 13 (43\%) pacientes la lactancia fue mixta.

Según la OMS, solo $35 \%$ de los lactantes de todo el mundo son alimentados exclusivamente con leche materna durante los primeros cuatro meses de vida(13) lo que concuerda en nuestro estudio donde el $40 \%$ de los niños recibieron lactancia materna de manera exclusiva.

Las madres de niños que nacieron por cesárea, pueden dar la lactancia materna sin inconvenientes $^{(14)}$ aunque en nuestro estudio observamos que el tener un parto vaginal o por cesárea no influyo en la forma de alimentación del menor ya que el $36 \%$ de las que dieron a luz por vía vaginal y $37 \%$ vía cesárea brindaron lactancia materna exclusiva. El hecho de que se hospitalizara al recién nacido si influyó ya que en un $75 \%$ de ellos se le introdujo formulas a su egreso.

Los motivos de abandono precoz de la lactancia materna en los menores de 6 meses encontrados en este estudio, coinciden con los de otras investigaciones ${ }^{(15)}$ donde se refiere la hipogalactia como la causa más frecuente en un $50 \%$.

Literatura revisada sugieren que muchas adolescentes abandonaron la lactancia cuando los niños eran menores de un mes de edad ${ }^{(16)}$ de la misma manera en nuestra investigación donde obtuvimos que la mitad de adolescentes alimenta a sus hijos con fórmula.

Indistintamente del nivel de educación materna se observaron prácticas de alimentación similares, diferente a lo observado por otros autores: madres con mayor nivel educativo seguían más frecuentemente las prácticas recomendadas. ${ }^{(17)}$

El trabajo o estudio materno aparece en una muy baja proporción como causa de destete, ${ }^{(18)}$ aunque en nuestro estudio solamente 3 (10\%) madres trabajaban pero es importante destacar que la totalidad de ellas practicaba la lactancia mixta.

Respecto al hecho de presentar enfermedad por problemas gastrointestinales observamos que de los niños alimentados con lactancia materna exclusiva el $58 \%$ presentaron proble- 
mas gastrointestinales pero a diferencia de los que se alimentaban con formula ninguno de estos requirió hospitalización por dicho problema, al contrario de lo que paso en los niños que recibieron lactancia mixta que probablemente perdieron su inmunidad ya que el $50 \%$ fueron ingresados por presentar cuadro entérico aunque no se realizaron exámenes inmunológicos.

Es de destacar que muchos niños se incorporan a la mesa familiar desde los 5 meses de edad ${ }^{(19}$ ${ }^{20)}$ algo que difiere en nuestro estudio ya que el $56 \%$ iniciaron su ablactación a los 6 meses y solo un $12 \%$ lo hizo antes de los 4 meses.

En conclusión, la lactancia materna exclusiva es la forma ideal para alimentar al recién nacido y lactante durante los primeros 6 meses de vida, la madre sabe esto pero hay algunos factores como ser la hipogalactia, hospitalizaciones al nacer, madres que trabajan y el ser adolescente que impiden que sea de manera exclusiva.

A pesar de que hace años se aplican programas con el objetivo de promover la lactancia materna exclusiva, la población desconoce y/o no entiende el significado de este término por lo que es necesario incrementar las acciones de salud con vistas a fortalecer los factores que promueven lactancia materna y disminuir los motivos de destete precoz. Proporcionar información sobre las técnicas de extracción manual, conservación y transporte de la leche materna, eliminando los biberones, por si trabaja o desea salir de casa.

\section{CONFLICTO DE INTERESES:}

Los autores manifiestan que durante la planeación y ejecución del presente trabajo de investigación, no se presentó ningún conflicto de interés.

\section{BIBLIOGRAFÍA}

1. Gamboa E, López B, Prada G y Gallo K. Conocimientos, actitudes y prácticas relacionadas con la lactancia materna en mujeres de edad fértil en una población vulnerable. Rev. chil. nutr. [online]. 2008 [citado 15 de junio 2015]; 35(1): 43-52. Disponible en: $<$ http://www.scielo.cl/scielo.php?script=sci_arttext\&pd=S0717-7518200800010000 6\&lng=es \&nrm =iso>. ISSN 0717-7518. http://dx.doi.org/10.4067/S0717-75182008 000100006.

2. Pino V, López E, Medel I, Andrea Pilar y Ortega S. Factores que inciden en la duración de la lactancia materna exclusiva en una comunidad rural de Chile. Rev. chil. nutr. [online]. 2013[citado 16 junio 2015]; 40 (1): 48-54. Disponible en: <http://www.scielo. cl/scielo.php?script $=$ sci_arttext $\&$ pid $=$ S0717-75182013000100 008\&lng=es\&nr $\mathrm{m}=$ iso > . ISSN 0717-7518. http://dx.doi.org/ 10.4067/S0717-75182013000100008.
3. Gonzales, Teresita; Escobar, Leticia; Gonzales, Luz. Prácticas de alimentación infantil y deterioro de la lactancia materna en México. Salud pública Méx [online].2013[citado 01 de junio 2015]; 55(2): 170-179. Disponible en: <http:// www.scielo.org.mx/ scielo.php?script=sci_arttext\&pid=S0036$36342013000800014 \&$ lng $=e s \& n r m=i s o>$. ISSN 0036-3634.

4. León, Hilda. Causas de abandono de lactancia materna en un hospital de tercer nivel. Pediatría de México 2010; 12(1):29-32.

5. Raventos, A. Prevalencia de la lactancia materna en el Área Básica de Salud Sant Feliu-2 (Barcelona). Rev Pediatr Aten Primaria. 2006; 8(31): 409-20.

6. Rodríguez J y Acosta N. Factores Asociados a la Lactancia Materna Exclusiva en Población Pobre de Áreas Urbanas de Colombia. Rev. salud pública 2008; 10(1):71-84. 
7. Nino M, Silva E, y Atalah S. Factores asociados a la lactancia materna exclusiva. Rev. chil. pediatr. [online]. 2012[citado 10 de junio 2015]; 83(2): 161-169. Disponible en: <http://www.scielo.cl/scielo. php?script= sci_arttext\&pid=S0370-4106201200020 0007\&lng=es\&nrm=iso $>$.ISSN 0370-4106. http://dx.doi.org/10.4067/S0370-41062 012000200007.

8.

Gonzalez I, Huespe M y Auchter M. Lactancia Materna Exclusiva Factores de Éxito y/o Fracaso. Revista de Posgrado de la VI Cátedra de Medicina. 2008; 177:1-5.

9.

Pinilla E, Orozco L, Camargo F, Peña E y Villabona N. Lactancia materna ineficaz: prevalencia y factores asociados. Revista Salud Uis, 2011; 43(3): 271-279.

10. Macias S, Rodriguez S y Ronayne P.Feeding pattern and incorporation of complementary foods in infants from a low- income population. Rev. chil. nutr. [online]. 2013[citado 19 junio 2015]; 40(3):235-242. Disponible en: <http://www. scielo.cl/scielo.php?script $=$ sci_arttext\&pid=S0717-7518201 $3000300004 \&$ Ing $=$ es\&nrm=iso $>$. ISSN 071 7-7518. http://dx. doi.org/10.4067/S071775182013000300004.

11. Madrigal H y Chias L. Lactancia materna, recomendación universal, y controversias en su aplicación en diferentes contextos socioeconómicos. Rev Esp Nutr Comunitaria 2010; 16(3):154-159.

12. Silva G. Leche materna y lactancia, siempre actual. CCAP 2012; 11 (3): 5-20.

13. Camargo F, Latorre J y Porras J. Factores asociados al abandono de la Lactancia Materna Exclusiva. Hacia la Promoción de la Salud. 2011; 16(1): $56-72$.

14. Organización Panamericana de la Salud."La alimentación del lactante y del niño pequeño: Capítulo Modelo para libros de texto dirigidos a estudiantes de medicina y otras ciencias de la salud". Washington, D.C.: OPS, (c) 2010 .

15. Avalos M. Comportamiento de algunos factores biosociales en la lactancia materna en los menores de un año. Rev Cubana Med Gen Integr [online]. 2011 [citado 05 de junio 2015]; 27(3): 323-331.

16. Quezada C, Delgado A y Arroyo L. Prevalencia de lactancia y factores sociodemográficos asociados en madres adolescentes. Bol Med Hosp Infant Mex. 2008; 65(2): 19-25.

17. Gatica C y Mendez M. Prácticas de alimentación en niños menores de 2 años. Arch. argent. pediatr. [online]. 2009[citado 20 junio 2015]; 107(6):496-503 . Disponible en: <http://www.scielo.org.ar/scielo.php? script $=$ sci_arttext\&pid $=$ S0325-0075200 9000600005\&lng=es\&nr m=iso>. ISSN 166 8-3501.

18. Nino R, Silva G y Atalah E. Factores asociados a la lactancia materna exclusiva. Rev Chil Pediatr 2012; 83(2): 161-169.

19. Cardoso K y et al. Introducción Precoz de Sustitutos de Lactancia Materna e Incidencia de Lactancia Materna Exclusiva al Mes de Vida. Rev. chil. pediatr. [online].2010[citado 19 junio 2015]; 81(4): 326-332. Disponible en: <http://www. scielo.cl/scielo.php? script=sci_arttext \&pid=S0370-410620100 00400006\&lng=es\&nr m=iso >. ISSN 03704106. http://dx.doi.org/10.4067/S0370-410 62010000400006.

20.

Caballero, $V$ y et al. Factores contribuyentes al abandono de la lactancia materna exclusiva en un área de salud. MEDISAN [online]. 2013[citado 18 junio 2015]; 17(3): 455-461. Disponible en:http://s cielo.sld.cu/scielo.php?script=sci_arttext\&pid=S1029-3019 2013000300005\&lng =es\&nrm=iso >. ISSN 1029-3019. 